\title{
Systemic Lupus Erythematosus Presenting With Posterior Reversible Encephalopathy Syndrome: An Unusual Case
}

\author{
Tanmayjyoti Sau ${ }^{1}$, Atri Chatterjee ${ }^{2}$, Sandip Kumar Dey ${ }^{3}$, Avishek Roy ${ }^{4}$ \\ ${ }^{I}$ (Associate Prpfessor, Department of Medicine, Nil Ratan Sircar Medical College, Kolkata, India) \\ 2,3,4 (Junior Resident, Department of Medicine, Nil Ratan Sircar Medical College, Kolkata, India)
}

\begin{abstract}
Posterior reversible encephalopathy syndrome (PRES) has been reported in association with hypertension, renal failure, transplantation and immunosuppressives [1]. However, reports of PRES in conjunction with SLE are sparse [2]. Majority of these patients had lupus nephritis and hypertension [3]. Recent reports have shown a stringent association of PRES with hypertension in SLE patients and response to cyclophosphamide-methylprednisolone pulse therapy [4]. Here, we report a rare case of a normotensive patient of SLE presenting with PRES. The patient was treatment-naïve and had significant renal involvement. Her neurologic impairment and overall disease activity improved following treatment with pulse doses of immunosuppressives.
\end{abstract}

Keywords - Lupus, Seizure, Posterior reversible encephalopathy Syndrome.

I.

Introduction:

Posterior reversible encephalopathy syndrome (PRES) has been reported in association with hypertension, renal failure, transplantation and immunosuppressives [1]. However, reports of PRES in conjunction with SLE are sparse [2]. Majority of these patients had lupus nephritis and hypertension [3]. Recent reports have shown a stringent association of PRES with hypertension in SLE patients and response to cyclophosphamide-methylprednisolone pulse therapy [4]. Here, we report a rare case of a normotensive patient of SLE presenting with PRES. The patient was treatment-naïve and had significant renal involvement. Her neurologic impairment and overall disease activity improved following treatment with pulse doses of immunosuppressive combination.

\section{Case Report:}

A 15 year old girl was admitted with history of generalized seizures for last six hours. There were eight episodes in total, each of them being tonic-clonic in nature, as per her parents' description. There was no prior history of seizures, fever, head trauma, headache or visual symptoms. The patient was conscious, but confused. Her vitals were: pulse 100/min, regular, blood pressure $130 / 76 \mathrm{~mm} \mathrm{Hg}$, respiration rate 22/min and temperature $98^{\circ} \mathrm{F}$. She had pallor and bilateral pedal edema. However, there were no focal neurodeficit.

The patient's seizures were controlled with intravenous phenytoin $(15 \mathrm{mg} / \mathrm{kg})$. A complete hemogram revealed normocytic normochromic anemia with elevated ESR. Total leukocyte count and differential were normal. Blood biochemistry revealed normal electrolytes, normal liver function tests except hypoalbuminemia. Urea and creatinine were slightly elevated. Cerebrospinal fluid study revealed a cell count of 20/ high power field (HPF), 90\% lymphocytes, protein $60 \mathrm{mg} / \mathrm{dl}$, glucose $40 \mathrm{mg} / \mathrm{dl}$. MRI of brain showed bilateral diffusion restricted hyperintense lesions in T2 and T2FLAIR sequences affecting cortical and subcortical white matter of fronto-parietal and occipital regions, suggestive of acute demyelination.

To investigate the cause of edema, routine examination of urine was done, which revealed 3+ albuminuria, 2-3 RBCs/HPF and RBC casts. Ultrasonography of abdomen showed increased cortical echogenicity of both the kidneys with mild ascites and minimal bilateral pleural effusion. Antinuclear antibody by indirect immunofluorescence was positive (1:160) and anti-ds DNA antibody was also positive (1:320).

Considering all these evidence, the patient was diagnosed as a case of SLE with nephritis. Renal biopsy confirmed presence of WHO class III disease. The pattern of demyelination was suggestive of PRES. The patient was started on immunosuppressive therapy consisting of methylprednisolone (1gm) and cyclophosphamide $\left(500 \mathrm{mg} / \mathrm{m}^{2}\right.$ of body surface area). The patient remained asymptomatic after the first pulse. She was discharged and asked to follow up after 3 weeks with a repeat MRI of brain. The repeat scan showed complete resolution of previous lesions, confirming the diagnosis of PRES. Repeat estimation of anti-ds DNA antibody revealed a titer of less than 1:80. She was scheduled for six pulses at one month interval. Till date the patient remains free of further neurological symptoms or signs. 


\section{Discussion}

PRES has been related to presence of hypertension and nephritis in SLE patients. Our case demonstrates that such patients may remain normotensive at presentation. The course of PRES does not parallel that of the disease. Though PRES has been reported early in the course of SLE, a patient presenting with PRES is unusual. In our patient, the cerebral lesions additionally involved subcortical structures.

There is no international guideline regarding treatment of PRES. Reports mention use of different regimens including cyclophosphamide alone, or in combination with plasmapheresis [5]. Those who developed PRES while on immunosuppressant discontinued the drug. In presence of major organ involvement, high dose immunosuppresants are needed and they do not adversely affect the outcome of PRES. We used a combination of methylprednisolone with cyclophosphamide. The patient responded to the regimen after the first pulse. Further pulses were scheduled due to the presence of nephropathy. However, it is not yet established the number of pulses required to treat PRES and whether intense therapy can prevent relapse.

\section{Figures}

Figure 1. MRI of brain (T2) showing extensive demyelinating lesions (arrows) at presentation (A-B), which completely resolved (C-D) after first pulse of immunosuppresants.
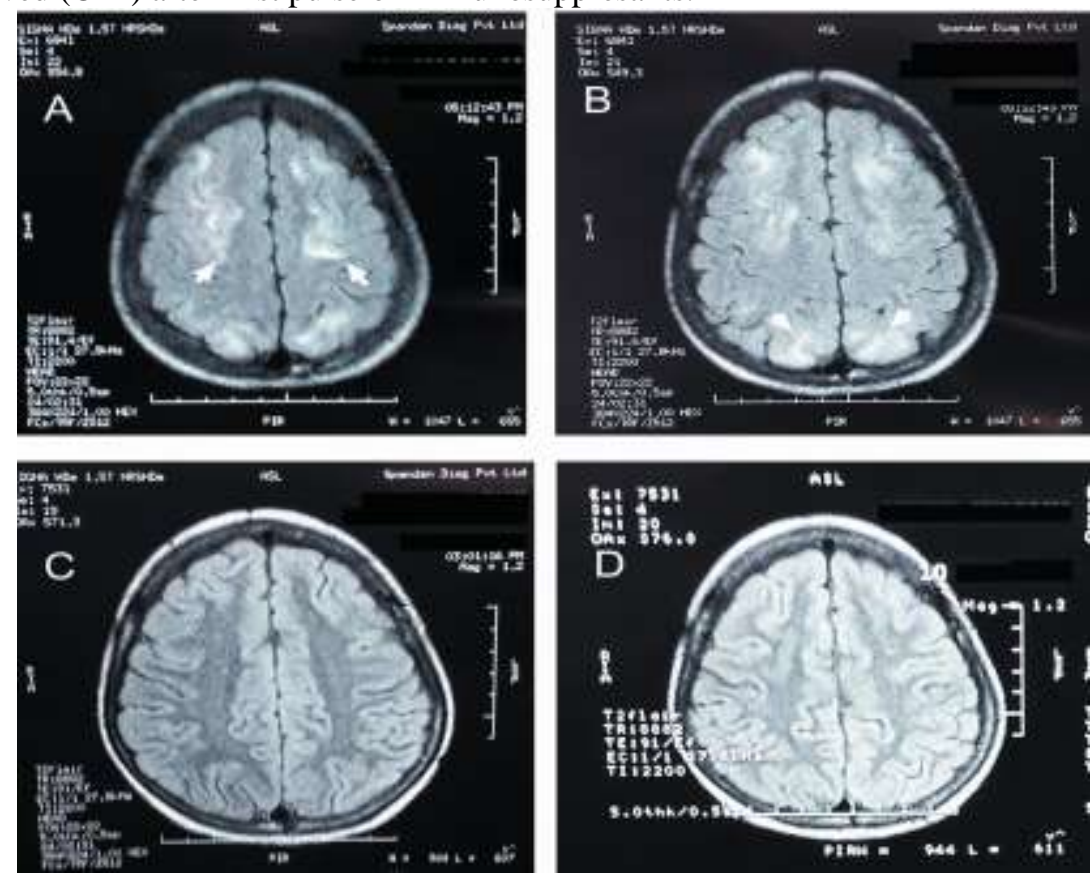

V.

Conclusion

In conclusion, we report a normotensive patient of SLE who first presented with PRES and recovered with a regimen of methylprednisolone-cyclophosphamide combination therapy. In absence of established guidelines of treatment of the condition, we plan to evaluate our regimen in further studies.

\section{REFERENCES}

[1] V. H. Lee, E. F. Wijdicks, E. M. Manno, A. A. Rabinstein, Clinical spectrum of reversible posterior leukoencephalopathy syndrome. Arch Neurol, 65(2), 2008, 205-210.

[2] M. L. Ishimori, B. D. Pressman, D. J. Wallace, M. H. Weisman, Posterior reversible encephalopathy syndrome: another manifestation of CNS SLE? Lupus, 16(6), 2007, 436-443.

[3] G. Leroux, J. Sellam, N. Costedoat-Chalumeau, D. Le Thi Huong, A. Combes, N. Tieulié, J. Haroche, Z. Amoura, A. Nieszkowska, J. Chastre, D. Dormont, J. C. Piette JC, Posterior reversible encephalopathy syndrome during systemic lupus erythematosus: four new cases and review of the literature, Lupus, 17(2), 2008, 139-147.

[4] I. R. Varaprasad, S. Agrawal, V. N. Prabu, L. Rajasekhar, M. A. Kanikannan, G. Narsimulu, Posterior reversible encephalopathy syndrome in systemic lupus erythematosus, J Rheumatol, 38(8) , 2011, 1607-1611.

[5] __. K. Kur, J. M. Esdaile. Posterior reversible encephalopathy syndrome--an underrecognized manifestation of systemic lupus erythematosus, J Rheumatol, 33(11), 2006, 2178-2183. 